\title{
A Study on Relationship of Endometrial Thickness and Abnormal Uterine Bleeding in Perimenopausal Women
}

\author{
1Jyoti Jaiswal, ${ }^{2}$ Anand Jaiswal, ${ }^{3}$ Richa Mishra
}

\begin{abstract}
Aims and objectives: To study the possible relationship between abnormal uterine bleeding (AUB) and endometrial thickness in perimenopausal women.
\end{abstract}

Materials and methods: A prospective observational study conducted on 60 cases of AUB in perimenopausal age (41-55 years) group.

Results: Institutional incidence of AUB in general and AUB in perimenopausal age was $10.7 \%$ and $29.38 \%$ respectively. Maximum cases were para 3 and of age $41-45$ years $(78.33 \%)$. Cases presented with irregular and heavy menstrual bleeding $(76.66 \%)$. About one-third $(33.33 \%)$ of the study subjects were suffering from severe and life-threatening anemia due to AUB. $90 \%$ study subjects required a blood transfusion, maximum of them were transfused preoperatively. As per PALM-COEIN classification, maximum cases had leiomyoma (41.66\%) as a structural cause of AUB followed by malignancy and hyperplasia $(33.33 \%)$. Majority of cases (45\%) presenting with AUB had an endometrial thickness between $6-9 \mathrm{~mm}$. Mean endometrial thickness was maximum in case of AUB M that is $10.33 \mathrm{~mm}$ followed by AUB-L $7.56 \mathrm{~mm}$, and AUB-A $7.2 \mathrm{~mm}$. Majority of cases $(70 \%)$ with ET $9 \mathrm{~mm}$ or less were having mild to moderate blood loss. Majority of cases with severe blood loss higher endometrial thickness; half subjects had ET $>10 \mathrm{~mm}$, and few had ET 16 to $21 \mathrm{~mm}$. Cafeteria approach for choosing the management line was given. Most of the perimenopausal cases preferred the surgical line of management $(83.33 \%)$ over medical method $(13.33 \%)$ despite counseling.

Conclusion: Endometrial thickness of more than $7 \mathrm{~mm}$ remains a common feature with estrogen-dependent conditions like fibroid, adenomyosis, hyperplasia, and malignancy even during perimenopause.

Clinical Significance: AUB in perimenopause needs vigilant surveillance, and endometrial thickness may be a relevant tool for it.

Keywords: AUB, Endometrial thickness, Perimenopause

How to cite this article: Jaiswal J, Jaiswal A, Mishra R. A Study on Relationship of Endometrial Thickness and Abnormal

${ }^{1}$ Professor , ${ }^{2}$ Associate Professor, ${ }^{3}$ Postgraduate Student

${ }^{1,3}$ Department of Obstetrics and Gynaecology, Pt. Jawahar Lal Nehru Memorial Medical College, Raipur, Chhattisgarh, India

${ }^{2}$ Department of Radiodiagnosis, Pt. Jawahar Lal Nehru Memorial Medical College, Raipur, Chhattisgarh, India

Corresponding Author: Anand Jaiswal, Associate Professor Department of Radiodiagnosis, Pt. Jawahar Lal Nehru Memorial Medical College, Raipur, Chhattisgarh, India, Phone: +919893231900, e-mail: dr.jyotijaiswal@gmail.com
Uterine Bleeding in Perimenopausal Women. J South Asian Feder Menopause Soc 2018;6(2):106-111.

Source of support: Nil

Conflict of interest: None

Date of received: 10 January 2018

Date of acceptance: 27 January 2018

Date of publication: July 2018

\section{INTRODUCTION}

The endometrium which lines the uterine cavity is one of the most dynamic tissues of the human body. A cyclic process of cell proliferation characterizes it, differentiation and death in response to sex steroids elaborated in the ovary. Abnormal uterine bleeding affects $10-30 \%$ of reproductive age group women and up to $50 \%$ of perimenopausal women. Pattern and causes of AUB differs in different age group and reproductive status of the woman. A variety of factors may cause abnormal uterine bleeding. The two most common causes are structural abnormalities of the reproductive system and ovulation disorder.

During perimenopause, shifts in hormone levels can affect ovulation and cause changes in the menstrual cycle. Because estrogen secretion continues, progesterone secretion declines, these hormonal changes can cause the endometrium to proliferate and increase the chance for polyps or fibroids that cause abnormal bleeding. Women in perimenopause are also at risk for other conditions that cause abnormal bleeding, including cancer, infection, and systemic illnesses, etc. Further evaluation is indicated, if a woman experiences persistent irregular menstrual cycles or an episode of profuse bleeding. Endometrial thickness determined by transabdominal/transvaginal sonography may influence early detection and treatment, and direct impact on prognosis by improving quality of life and decreasing morbidities and mortalities.This study was done to note the possible relationship between endometrial thickness and AUB in perimenopausal women.

\section{AIMS AND OBJECTIVES}

To study the possible relationship between abnormal uterine bleeding (AUB) and endometrial thickness in perimenopausal women. 


\section{MATERIALS AND METHODS}

The study is a prospective observational study conducted in the Department of the Obstetrics and Gynecology, Dr. BR Ambedkar Memorial Hospital Raipurfrom January 2016 to September 2016. Cases ofAUB in perimenopausal age who were willing to participate in the study were included by simple random sampling $(n=60)$.

\section{EXCLUSION CRITERIA}

- Pregnancy and pregnancy-related conditions.

- Females not in perimenopausal age group.

- Patients with a bleeding disorder.

- Patients with known liver/renal diseases.

- Endocrine disorders like hyper- or hypothyroidism.

- On medications like steroids, neuroleptics, anticoagulants and cytotoxic agents.

- Hormone-producing ovarian tumors in USG.

- IUCD.

- Recent uterine perforation.

- Patients with infectious causes

- Patients with neoplasia or CIN changes.

- Injuries of vulva and vagina.

Each woman underwent a transvaginal/transabdominal ultrasonic examination to estimate the endometrial thickness. Routine hemogram and other biochemical investigations were carried out as and when required. Statistical analysis was done by using descriptive and inferential statistical method.

\section{RESULTS}

The outcome of the study is as follows:

Total of 7125 patients attended the gynecology outpatient department ( OPD) in the year, out of which 769

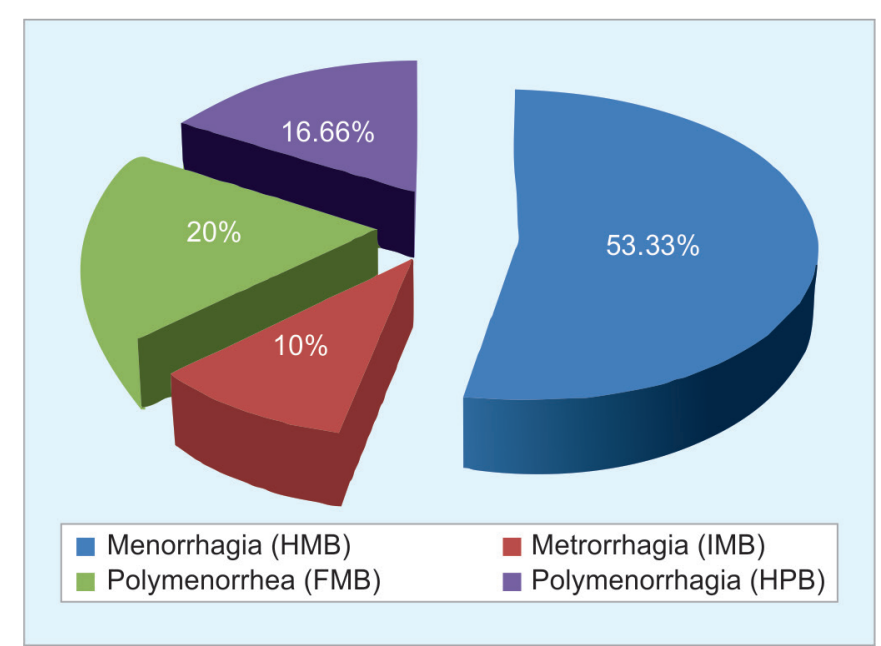

Graph 1: Distribution of cases according to bleeding pattern patients were suffering from abnormal uterine bleeding giving an institutional incidence of AUB as $10.7 \%$. Out of these cases, a total number of cases in the perimenopausal age group was 226. So the institutional incidence of AUB in perimenopausal age group was $29.38 \%$.

Maximum cases were para 3 of age 41 to 45 years (78.33\%). Cases had a history of irregular (41.66\%) and heavy $(76.66 \%)$ menstrual bleeding and a history of the passage of clots present in $63.3 \%$ of study subjects. The most common type of bleeding pattern was HMB (53\%) (Graph 1). About one-third (33.33\%) of the study subjects were suffering from severe, and life-threatening anemia due to AUB (Graph 2) 90\% study subjects required a blood transfusion, maximum of them were transfused preoperatively, for correction of anemia. On pelvic ultrasound average uterine volume was $85 \mathrm{cc}$ in AUB-P, 160cc in AUB-A, 248cc in AUB-L and 131.9cc in AUB-N (Table 1). As per Palm-Coein classification, maximum cases had leiomyoma (41.66\%) as a structural cause of AUB followed by malignancy and hyperplasia (33.33\%) (Graph 3).

Majority of cases (45\%) presenting with AUB had an endometrial thickness between 6 and $9 \mathrm{~mm}$ (Graph 4). Mean endometrial thickness was maximum in cases of AUB $\mathrm{M}$ that is $10.33 \mathrm{~mm}$ followed by AUB-L $7.56 \mathrm{~mm}$, and AUB-A $7.2 \mathrm{~mm}$ (Table 2). Majority of cases (70\%) with ET $9 \mathrm{~mm}$ or less were having mild to moderate blood loss. Majority of cases with severe blood loss had endometrial thickness $>7 \mathrm{~mm}(16 / 20$ cases, $80 \%)$, out of which half subjects had ET $>10 \mathrm{~mm}$, and few had ET 16-21 mm (Table 3). Most of the perimenopausal cases preferred the surgical line of management $(83.33 \%)$ over medical method (13.33\%) despite counseling.

\section{DISCUSSION AND REVIEW OF LITERATURE}

Abnormal uterine bleeding (AUB) is one of the most common presenting complaints encountered in a gyne-

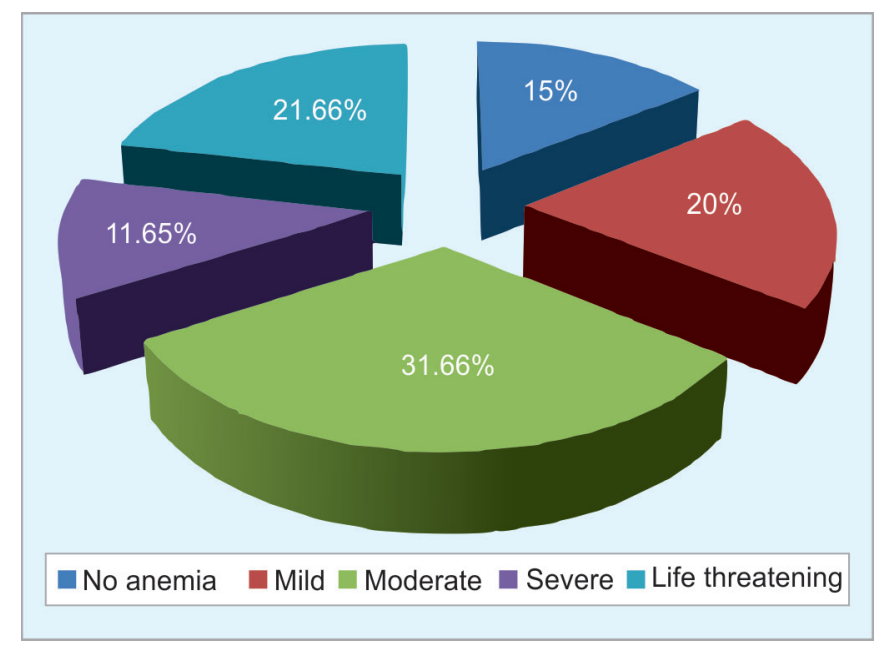

Graph 2: Distribution of cases according to severity of anemia 
Table 1: Distribution of cases according to uterine volume and type of AUB as per Palm-Coein classification

\begin{tabular}{|c|c|c|c|c|c|c|c|c|c|c|}
\hline UT. Vol & $A \cup B-P$ & $A \cup B-A$ & $A \cup B-L$ & AUB-M & $A \cup B-C$ & $A \cup B-O$ & $A \cup B-E$ & $A \cup B-I$ & $A \cup B-N$ & Total \\
\hline $51-75$ & 2 & - & - & 2 & - & - & - & - & - & 4 \\
\hline 75-100 & 1 & 1 & 2 & 6 & - & - & - & - & 1 & 11 \\
\hline $101-125$ & 1 & 2 & 5 & 3 & - & - & - & - & 2 & 13 \\
\hline $126-150$ & - & 2 & 5 & 6 & - & - & - & - & - & 13 \\
\hline $151-175$ & - & - & 1 & 1 & - & - & - & - & - & 2 \\
\hline 176-200 & - & 1 & 3 & - & - & - & - & - & - & 4 \\
\hline $201-250$ & - & 1 & 3 & 1 & - & - & - & - & - & 5 \\
\hline $251-300$ & - & - & 1 & - & - & - & - & - & - & 1 \\
\hline $\begin{array}{l}300 \text { and } \\
\text { above }\end{array}$ & 1 & - & 5 & 1 & - & - & - & - & - & 7 \\
\hline Total & 5 & 7 & 25 & 20 & - & - & - & - & 3 & \\
\hline Mean & 85.4 & 160 & 248.9 & 131.9 & & & & & 80.34 & \\
\hline
\end{tabular}

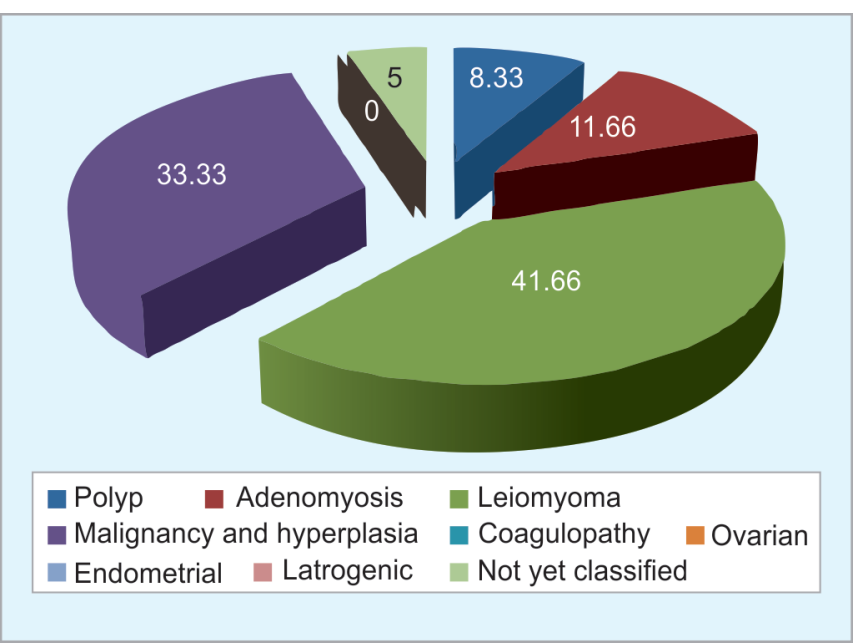

Graph 3: Distibution of cases as per Palm-Coein classification

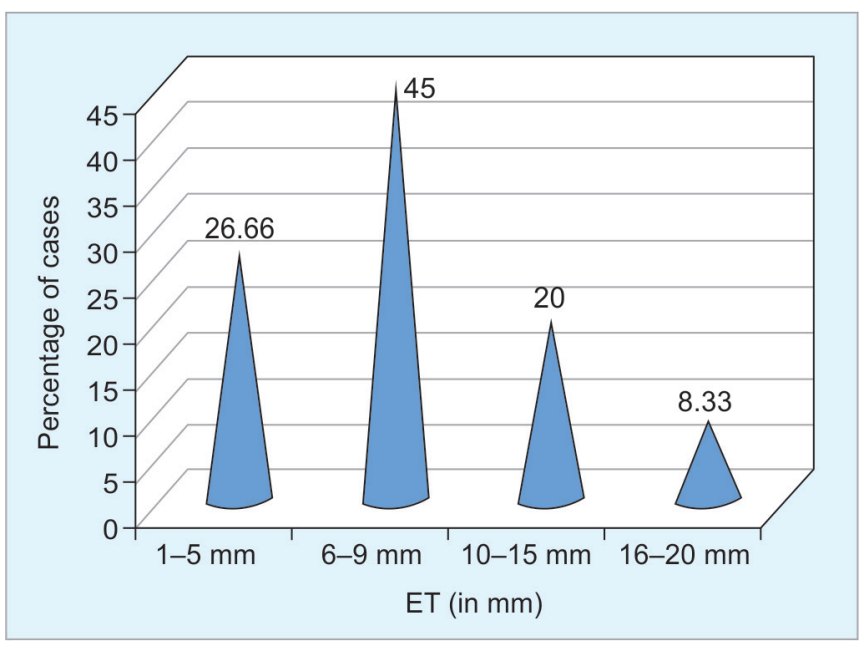

Graph 4: Distribution of cases according to endometrial thickness

Table 2: Distribution of cases according to endometrial thickness and type of AUB as per Palm-Coein classification

\begin{tabular}{lllllllllll}
\hline ET. thickness & AUB-P & $A U B-A$ & $A U B-L$ & $A U B-M$ & $A U B-C$ & $A U B-O$ & AUB-E & AUB-I & AUB- $N$ & Total \\
\hline $1-3$ & 0 & 0 & 1 & 1 & 0 & 0 & 0 & 0 & 0 & 2 \\
$4-6$ & 3 & 2 & 9 & 2 & 0 & 0 & 0 & 0 & 1 & 17 \\
$7-9$ & 1 & 3 & 8 & 9 & 0 & 0 & 0 & 0 & 2 & 23 \\
$10-12$ & 0 & 0 & 3 & 3 & 0 & 0 & 0 & 0 & 0 & 6 \\
$13-15$ & 0 & 1 & 4 & 0 & 0 & 0 & 0 & 0 & 0 & 5 \\
$16-18$ & 0 & 0 & 0 & 4 & 0 & 0 & 0 & 0 & 0 & 4 \\
$19-21$ & 1 & 0 & 0 & 1 & 0 & 0 & 0 & 0 & 0 & 2 \\
$>21$ & 0 & 1 & 0 & 0 & 0 & 0 & 0 & 0 & 0 & 1 \\
TOTAL & 5 & 7 & 25 & 20 & 0 & 0 & 0 & 0 & 3 & 6.6 \\
MEAN & 4.56 & 7.2 & 7.56 & 10.33 & 0 & 0 & 0 & 0 &
\end{tabular}

Table 3: Correlation of severity of AUB with endometrial thickness

\begin{tabular}{lllll}
\hline ET (in $\mathrm{mm})$ & Mild & Moderate & Severe & Total \\
\hline $1-3$ & 1 & 1 & - & 2 \\
$4-6$ & 3 & 10 & 4 & 17 \\
$7-9$ & 3 & 11 & 9 & 23 \\
$10-12$ & 1 & 1 & 4 & 6 \\
$13-15$ & 2 & 3 & 1 & 6 \\
$16-18$ & - & 2 & 2 & 4 \\
$19-21$ & - & 2 & - & 2 \\
$>21$ & - & - & - & - \\
\hline
\end{tabular}

cologist's office or primary care setting. An estimated $60 \%$ of visits to a physician's office are from women seeking treatment for menstrual problems. Unpredictable and unscheduled bleeding often lead to psychological, medical, and sexual problems requiring pharmacologic and surgical interventions. The reported prevalence of AUB in India is $17.9 \% 72$ In the present study, the institutional incidence of AUB was 10.7\%.

Women entering the perimenopausal age group (40-50) may have recurrent bouts of AUB and associated 
physical complaints due to changes in the hypothalamicpituitary-ovarian axis. The hormonal milieu is associated with decreased inhibin levels and variable estradiol levels, normal follicle stimulating hormone (FSH), and menstrual cycles that can be episodically ovulatory. Myriad menstrual complaints occur during the perimenopause, including menometrorrhagia, amenorrhea, and oligomenorrheic cycles.

Pillai ${ }^{1}$ have reported a higher incidence of AUB in the age group of 48-51 years (40\%). Similarly, Najeeb et al. ${ }^{2}$ in a study on 141 women with AUB maximum patients were between 40 years and 47 years age group with a mean age of 44 years. Sajitha et al., ${ }^{3}$ given a large range of 46-55 yearsage group (42.94\%) while Shobhitha et al. ${ }^{4}$ found the majority of patients of AUB in 41-50 years.

Parveen et al. ${ }^{5}$ found that approximately $45 \%$ of women in the age group of 40-50 years, followed by $40 \%$ in $30-40$ years age group, had abnormally thickened endometrium causing AUB.

Breitkopf et al. ${ }^{6}$ showed in 2006 that the incidence of bleeding increases with age and parity. Our study revealed that the occurrence of menstrual disorders increases with advancing age. A similar occurrence of AUB in this age group was reported by Yusuf et al. and Muzaffar et al. ${ }^{7}$ in their study.

The reason for the increased incidence of abnormal uterine bleeding in this age group may be because these patients are in their climacteric period. As women approach menopause, cycles shorten and often become intermittently anovulatory due to a decline in the number of ovarian follicles and the estradiol level. Cycles may also get prolonged resulting in an extended duration of amenorrhea followed by heavy bleeding.

Thakur et al. ${ }^{8}$ found that the incidence of AUB was higher in high parity women, as $72 \%$ were gravid III and above. Talukdar et al. ${ }^{9}$ showed that the incidence of bleeding increases with age and parity. AUB was seen in $42.71 \%$ cases in para 4 or more followed by para 3 (26.21\%). In the present study also maximum (75\%) patients were multiparous with parity 3 or more.

In perimenopausal AUB, bleeding is often noncyclic, variable in amount and volume, and unpredictable. In the present study, subjects had various patterns of menstrual bleeding. A total of 20 cases (33.33\%) had continuous bleeding, 15 cases $(25 \%)$ had regular pattern while maximum 25 cases $(41.66 \%)$ had a history of irregular and heavy bleeding. Various terms used for menstrual disorders used were-menorrhagia (HMB), metrorrhagia (IMB), polymenorrhea (FMB).

Shobhitha et al. ${ }^{4}$ suggested the incidence of menorrhagia as $40 \%$, followed by metropathia haemorrhagica $30 \%$, polymenorrhea $25.4 \%$. Katke et al. ${ }^{10}$ found that menorrhagia was the most frequent $(74.24 \%)$ followed by polymenorrhagia.

In the present study menorrhagia / HMB (53.3\%) was seen in maximum cases, followed by polymenorrhea / FMB (20\%), followed by polymenorrhagia/HPB (16.6\%) which was following the above mentioned studies.

To assess the menstrual blood loss in AUB cases is a subjective thing. What's heavy for one woman may be normal for other. By definition, heavy menstrual bleeding is defined as excessive menstrual blood loss which interferes with the woman's physical, emotional, social and marital quality of life. Heavy menstrual bleeding is considered when blood loss is $80 \mathrm{~mL}$ or more in each cycle. In the present study, about $50 \%$ of cases reported bleeding as moderate type while $33 \%$ had severe bleeding. Only $16.66 \%$ of the cases reported with mild bleeding.

The severity of anemias-AUB is a significant contributor for anemia after pregnancy-related blood loss. In the present study $90 \%$ study subjects required a blood transfusion for AUB, maximum of them (61.11\%) were transfused preoperatively, for correction of anemia.

In the present study, about one-third (33.33\%) of the study subjects were suffering from severe anemia due to AUB followed by a similar number of subjects with moderate anemia. Kristen et al. showed similar results.

Pelvic ultrasound is the least invasive of the outpatient techniques that can be used for visualization of the structure of the uterus, its volume and the details of the endometrium. It helps in the diagnosis of structural abnormalities as an explanation for AUB, such as submucous fibroids or endometrial polyps.

The uterine volume changes in different phasesof life according to hormone milieu. In prepuberty it is 10-20 $\mathrm{mL}$, in nulliparous women, it is $30-40 \mathrm{~mL}$, in multiparous women it is $60-80 \mathrm{~mL}$ while in postmenopausal women it is $14-17 \mathrm{~mL}$.

In the present study, on pelvic ultrasound maximum patients $(70 \%)$ had uterine volume $>100$ cc. AUB-L (41.6\%) was a most common presentation followed by AUB-M (33.33\%), AUB-A (11.66\%) and AUB-P (8.33\%) (Graph 3). Highest uterine volume was $758 \mathrm{cc}$ in a patient of leiomyoma. Highest mean volume was in AUB-L (248.9cc) followed by $160 \mathrm{cc}$ in AUB-A, 131.9cc in AUB-M, 85.4cc in AUB-P and 80.3cc in AUB-N (Table 1).

\section{Endometrial Thickness}

In perimenopausal age, the effect of unopposed estrogen causes endometrial proliferation and thickened endometrium. Role of endometrial thickness measurement is well established and a 4-5 mm cut off value has a high negative predictive value in excluding endometrial hyperplasia or cancer. Endometrial thickness is an indirect indicator of 
endometrial hyperplasia and amount of menstrual bleeding.In the present study ultrasonographic endometrial thickness was evaluated in all cases. Meta-analysis has demonstrated that sonographic measurement of endometrial thickness is an acceptable test for prediction of endometrial pathology, but it has limitations in correctly diagnosing the type of endometrial pathology.

In perimenopausal and postmenopausal women with abnormal bleeding, endometrial biopsy generally is considered unnecessary when the endometrial thickness is less than 4 or $5 \mathrm{~mm}$ because the risk of endometrial hyperplasia or cancer is remote. But as the endometrial thickness increases chances of hyperplasia with atypia and malignancy increases proportionately.

Endometrial thickness and severity of bleeding: In the present study correlation of endometrial thickness and severity of bleeding was tabulated. Out of 60cases, 10 had mild, 30 had moderate, and 20 had severe bleeding. Observations suggested that majority of cases, 7/10 (70\%) who were having mild blood loss were having endometrial thickness $9 \mathrm{~mm}$ or less. 22/30 (73.3\% ) cases with moderate blood loss were also having an endometrial thickness up to $9 \mathrm{~mm}$.

While most of the cases $(86.6 \%)$ in the severe blood loss group had ET $7 \mathrm{~mm}$ and more even with continuous bleeding episodes and $13.3 \%$ of cases were having a very high endometrial thickness of 16-21 mm (Table 3).

In the present study endometrial thickness (6 to $9 \mathrm{~mm}$ ) was found in a maximum number of patient $(45 \%)$ followed by 1 to $5 \mathrm{~mm}$ in $26.6 \%$ of the study subjects. This finding could be because most of the patients presented to us after an episode of heavy bleeding that has resulted in shedding of endometrium before an ultrasonographic examination. The other factor may be that not only endometrial thickness but other factors like the area of the endometrium and its vascularity may also be responsible for heavy bleeding

Najeeb et al. ${ }^{2}$ studied AUB in 77 perimenopausal women and found thatendometrial thickness of more than $8 \mathrm{~mm}$ is considered suspicious of endometrial pathology in perimenopausal women with AUB and further investigation is recommended.

Minimum endometrial thickness considered to be abnormal was accepted as $5 \mathrm{~mm}$ in a study conducted by Dasgupta et al. ${ }^{11}$ In a study conducted by Shobhitha et al. ${ }^{4}$ an endometrial thickness of $8 \mathrm{~mm}$ or more can be further subjected to dilatation and curettage as this study showed that with an endometrial thickness cutoff of $8 \mathrm{~mm}$ the dilatation and curettage can be avoided in $40 \%$ women.

In the present study, the endometrial thickness was tabulated according to the type of pathology related to AUB as per Palm-Coein classification also and it was observed that mean endometrial thickness was highest in cases with AUB-M (10.33 mm), followed by AUB-L (7.56 mm), followed by AUB-A $(7.2 \mathrm{~mm})$; as these are estrogen dominant conditions (Table 2).

In a study conducted by Katke et al. ${ }^{10} 43.9 \%$ of the total patients were having fibroid as associated pathology on ultrasound. It was also found that all cases of AUB with uterine fibroids had an endometrial thickness of $>10 \mathrm{~mm}$ on ultrasound, suggestive of endometrial hyperplasia. In our study also mean endometrial thickness among patients with fibroids was $7.56 \mathrm{~mm}$ (Table 2).

\section{Type of Management}

There are various methods for managing AUB depending on the cause related to it. Management includes medical methods, conservative surgical techniques and definitive surgeries including hysterectomy. Therapy aims to reduce blood loss, reduce the risk of anemia and to improve quality of life. While planning the management of the case, the factors taken into consideration were age, the severity of bleeding and the patient's interest to retain fertility.

Medical management: Medical therapy is now the mainstay of treatment of AUB. It is usually given to women who wish to retain her fertility or the cases who are not willing for removal of the uterus, subjects who are waiting for surgery and in whom the prognosis with the medical method is fairly good. The different types of medical therapies used for the treatment of AUB are NSAIDS, antifibrinolytics, progestins, COCs, danazol, and GnRH, etc.

ACOG recommends treatment with combination oral contraceptives or cyclic progestin for anovulatory uterine bleeding.

\section{Surgical management}

\section{Minimal Invasive Surgeries}

Newer techniques were developed immensely over the last two decades to accomplish endometrial destruction as a treatment option for women with AUB and other forms of intractable uterine bleeding who wish to retain their uteri and not interested in future childbirth.

\section{Hysterectomy}

About two-thirds of all hysterectomies worldwide are done in perimenopausal age group ( $40-45$ years) due to AUB as hysterectomy offers women with AUB a definitive solution and is known to have high rates of patient satisfaction.

Though hysterectomy is a most effective method it should be avoided in younger cases as various studies suggest that ovarian function diminishes after hysterectomy and leads to problems associated with it. Hys- 
terectomy can be recommended for heavy menstrual bleeding. This only should be considered for patients who no longer desire childbearing and who have failed other hormonal and/or surgical treatments and who have been thoroughly evaluated.

Removal of the uterus is the most effective treatment for bleeding and overall satisfaction rates approximate $85 \%$. Disadvantages include an increased number of adverse effects, longer recovery time and higher initial care costs compared with uterine-sparing procedures. Hysterectomy also may be associated with ovarian failure nearly four years earlier than expected.

Nearly $83.33 \%$ cases in the present study preferred the surgical line of management over medical method $(13.33 \%)$, as most of the cases recruited in the study were above 40 years of age, who needed admission, blood transfusion and were not satisfied with medical management or not willing for conservative management and follow-up. They were from the peri-menopausal age group in whom family was already completed and were willing for hysterectomy as definitive management.

In a randomized controlled trial by Pinion et al. ${ }^{12}$ comparing hysterectomy with TCRE for AUB concluded that hysteroscopic endometrial ablation was superior to hysterectomy regarding operative complications and postoperative recovery, but satisfaction after hysterectomy was significantly higher.

\section{CONCLUSION}

Abnormal uterine bleeding is a common and sometimes debilitating condition in women of perimenopausal age. Endometrial thickness assessment is mandatory while planning for management of AUB.

AUB has a direct association with endometrial thickness. Endometrial thickness of more than $7 \mathrm{~mm}$ remains a common feature with estrogen-dependent conditions like fibroid, adenomyosis, hyperplasia, and malignancy even during perimenopause. Increased endometrial thickness leads to severe blood loss also.

\section{REFERENCES}

1. Pillai SS. Sonographic and histopathological correlation and evaluation of endometrium in perimenopausal women with abnormal uterine bleeding. Int J Reprod Contracept Obst Gynecol. 2014 Mar;3(1):113-117.

2. Najeeb R, Awan AS, et al. Role of transvaginal sonography in assessment of abnormal uterine bleeding in perimenopausal age group. J Ayub Med Coll Abbottabad 2010;22(1):87-90.

3. Sajitha K. Study of histopathological pattern of endometrium in abnormal uterine bleeding CHRISMED. J Health Res 2001; 1(2):76-81.

4. Shobhitha GL, Kumari VI, et al. Endometrial study by TVS and It's correlation with histopathology in abnormal uterine bleeding. J Dental Med Sci 2015;14(4):21-32

5. Parveen N, Majeed R, et al. Attitude and knowledge of medical students of Isra University about dysmenorrhoea and its treatment. J Ayub Med Coll Abbottabad 2009;21:159-162.

6. Breitkopf DM, Frederickson RA, et al. Detection of benign endometrial masses by endometrial stripe measurement in premenopausal women. Obstet Gynecol 2004;104(1):120-125

7. Muzaffar M, Akhtar K A, et al. Menstrual Irregularities with excessive blood logs: A clinicopathological correlation. J Pak Med Assoc 2005; 55: 486-489

8. Thakur S., Nayak SR, et al. Geriatric problem: Abnormal uterine bleeding per vaginum after the age of 40. J Obetetr Gynaecol India 1976;44(3):580-586.

9. Talukdar B, Mahela S. Abnormal uterine bleeding in perimenopausal women: Correlation with sonographic findings and histopathological examination of hysterectomy specimens. J Midlife Health 2016;7(2):73-77.

10. Katke RD, Zarariya AN. Use of diagnostic hysteroscopy in abnormal uterine bleeding in perimenopausal age group and its clinicopathological co-relation with ultrasound and histopathology findings: experience in a tertiary care institute. Int J Reprod Contracept Obstet Gynecol 2015;4(2):413-418.

11. Dasgupta S, Chakraborty B, et al. Abnormal uterine bleeding in perimenopausal age: Diagnostic options and Accuracy. J Obstet Gynecol India 2011;61(2):189-194.

12. Pinion SB, Parkin DE, et al. Randomised trial of hysterectomy, endometrial laser ablation, and transcervical endometrial resection for dysfunctional uterine bleeding. BMJ 1994;309:979-983. 